\title{
EFFICACY OF TWO DIFFERENT RECONSTRUCTION MODALITIES AFTER SURGICAL RESECTION OF MANDIBULAR FIBROUS DYSPLASIA (A CLINICAL STUDY)
}

\author{
Abdelbadia A. Abdelmabood*, Abdel Aziz B.Abdullah"** Hossam E. Mohamed ${ }^{* *}$ and Omar S.Ali***
}

\begin{abstract}
Objective: To evaluate efficacy of Nonvascularized bone graft ( NVBG) with bone marrow aspirate concentrate (BMAC) compared with free vascularized fibula graft in reconstruction of mandible after surgical treatment of monostatic mandibular fibrous Dysplasia.

Patients and methods: The present study was carried out of three years on 14 patients (8 males \& 6 female) ranging from 9- 18 years who had monostatic fibrous dysplasia and need segmental resection and immediate reconstruction. The patients were divided randomly into two equal groups; group I patients grafted with free vascularized fibula graft after segmental resection and group II patients grafted with iliac crest or rib bone graft and injected with BMAC at surgical site after radical excision of lesion. They were selected from those attending the out patient clinic at Faculty of Dental Medicine, Al Azhar University, Assuit branch and the Faculty of Dentistry, in coordination with Plastic Surgery at Suez Canal University Hospital over a period of 4 years (July 2013 -July 2017). Postoperative clinical, radiographic evaluation were performed at 6 month and 12 month till two years after that to assess recurrence of lesion and bone graft density.
\end{abstract}

Results: Both groups was clinically uneventful except two cases in group I and one case in group II .All patients had adequate facial contour, proper facial symmetry and good take post operatively. Radiographic evaluation showed at six month, there was a highly statistically significant difference between two groups. This means that, newly formed bone of group I was significantly denser than group II. At 12 months, the mean of the group I was more than the mean of group II . These showed a difference between means of two groups but this difference was not a statistically significant .

Conclusion: Conventional grafting techniques with NVBGs improved by BMAC injection with satisfactory outcomes. Thereby it can be used as alternative modality for free vascularized bone graft in mandibular reconstruction in cases with massive monostatic fibrous dysplasia depend on the size of the lesion

* Lecturer of Oral and Maxillofacial Surgery, Faculty of Dentistry, Suez Canal University

** Lecturer of Oral and Maxillofacial Surgery, Faculty of Dental Medicine (Assiut) - Al-Azhar University

*** Lecturer of Plastic Surgery, Faculty of Medicine, Suez Canal University 


\section{INTRODUCTION}

Fibrous dysplasia (FD) is a bone development anomaly characterized by hamartomatous proliferation of fibrous tissue within the medullary bone, with secondary bony metaplasia, producing immature, newly formed and weakly calcified bone, without maturation of the osteoblast which appears radiolucent on radiographs, with the classically described ground-glass appearance ${ }^{(1)}$.

FD are divided into about $70 \%$ in form of monostatic and about $30 \%$ as polystatic. Albright syndrome that characterized by association of FD and precocious puberty and skin pigmentation with some forms of endocrine abnormalities. Malignant transformation were rare about $0.5 \%$ for monostatic form and about $4 \%$ in Albright syndrome (2-5). Differential diagnosis of maxillofacial FD include Paget's disease, ossifying fibroma and sarcoma of the jaw. But the most useful diagnostic tool based on its specific radiographic ground glass appearance on the selected computed tomograhic (CT) ${ }^{(6)}$.

Although FD is slowly progressive disorder of the bone but there are associated complications due to rapid replacement of cancellous bone by immature woven bone and fibrous tissue. This high production of immature bone lead to associated complications as pain, osteoarthritis, deformities , pathological fractures and nerve compression. But in fact FD tend to be self limiting after puberty ${ }^{(7-9)}$.

Treatment of maxillofacial FD depends mainly on patient age , associated complications , extended size of the lesion and site. However the complex 3 dimension of the skull and face with the complicated anatomy may be difficult to recontour this region ${ }^{(10)}$. Surgical corrections recommended to rebuild resected part of bone to avoid its relapse ${ }^{(11)}$.

This can be achieved through a variety of surgical techniques, mainly non-vascularized and vascularized grafts. Nonvascularized bone grafts
(NVBGs) may be suitable in specific cases. But ,there are several drawbacks for NVBGs such as donor site complications, and lack of blood supply results in slow and incomplete healing, increased rates of infection, non-union, and fracture as well as rapid resorption of bone graft along time ${ }^{(12,13)}$.

With advancement of microvascular surgery, vascularized bone grafts (VBGs) contain an intrinsic blood supply that adds the biological advantage of shortened union time as well as superior functional and aesthetic scores for diet, speech, and midline symmetry. Outcomes from free VBGs, most notably free fibula grafts, have proved markedly superior to non-vascularized options, including reconstruction plates and bone grafts, with defects of the mandible ${ }^{(12-14)}$.

Recently, Future strategies include tissue engineering approaches utilizing collagen-based scaffolds combined with bone marrow-derived stromal cells and growth factors ${ }^{(15,16)}$. Furthermore, bone marrow stem cells (BMSC) and plateletrich plasma (PRP) has been shown to form microstructures of the cellular bone resembling those in normal mandibles ${ }^{(17) .}$ Although ,there are several drawbacks for BMSC usage such as culture expansion technique which requires additional visit to application, special equipment, and additional training to harvest the stem cells. So that, recent investigations uses bone marrow aspirate concentrate (BMAC) to avoid time-consuming and technically difficult process of cell expansion and differentiation of BMSC ${ }^{(18)}$.

Nevertheless, none of these approaches can yet be considered established as standard in craniomaxillofacial reconstructive surgery. Therefore, the present study was designed to assess NVBGs with BMAC compared with free vascularized fibula graft in reconstruction of mandible after surgical treatment of monostatic mandibular fibrous Dysplasia. 


\section{PATIENTS AND METHODS}

\section{Patient selection :}

The present study was carried out three years on 14 patients who had monostatic fibrous dysplasia through. Eight of these patients were males and six were females. The average patient age at the time of surgery was 13.5 years (ranging from 9- 18 years).

They were selected from those attending the out patient clinic at Faculty of Dental Medicine, $\mathrm{Al}$ Azhar University, Assuit branch and the Faculty of Dentistry, in coordination with Plastic Surgery at Suez Canal University Hospital over a period of 4 years (July 2013 -July 2017). They were informed of the need to attend 3 years of follow-up. The patients were required to provide informed consent or to refuse participation in the study.

\section{Inclusion criteria:}

- Massive monostatic mandibular fibrous dysplasia.

- Absence any Syndromic features or osseous malformations of the face in the extended family

- There was also no trauma history in any patient.

- Preoperative radiographic presented fibrous dysplasia as poorly defined ovoid areas of dysplastic bone that exhibited a "ground glass" appearance; this was suggestive of a diagnosis of fibrous dysplasia.

- The preliminary biopsy before surgery revealed fibrous dysplasia in all patients.

- Absence of any blood disorders which cause complications after the bone marrow aspiration procedures

- Absence of any systemic disorders that may influence the course if wound healing

\section{Patients grouping:}

The patients were divided randomly into two equal groups:

Group I : Included 7 patients grafted with free vascularized fibula graft. Group II: Included 7 patients grafted with iliac crest or rib bone graft and injected with BMAC at surgical site.

\section{Preoperative procedures:}

Preoperative procedures included case history, intraoral and extra oral clinical examination, and radiographic examination by orthopantomograph and CT scan and finally, surgical biopsy to investigate fibrous dysplasia cases.

\section{Surgical operation :}

Patients were prepared for surgery with the basic traditional method. All operation were done under general anesthesia. Segmental mandibulectomy with insertion of half titanium reconstruction plate was performed with simultaneous vascularized fibula graft alone in group I (fig. 1) and iliac crest or rib bone grafting with injection of BMAC in group II.

\section{Preoperative measurements prior to free vascu- larized fibular graft:}

- Preoperative evaluation is undertaken prior to performing fibular vascularized tissue flap. A complete medical history and physical exam are undertaken, including laboratory and imaging studies as indicated.

- Specialized equipment is required to perform microvascular fibular graft procedures. Includes microvascular surgical instruments, microsurgical anastomotic couplers, surgical loupes, an operating microscope, microsurgical sutures and vascular clamps, Doppler ultrasound (external and internal), and warmed, heparinized irrigation fluids.

- A typical microsurgical instrument set consists of multiple jeweler's forceps (curved and straight), microsurgical adventitial scissors (curved and straight), microsurgical suture scissors, vein dilator forceps, vascular clamp applying forceps, and microsurgical needle holders.

- The vascular clamp set contains multiple arterial and venous, single and double approximating clamps with or without frames of varying size. Venous anastomotic coupler devices are being used routinely 


\section{Surgical procedure for harvesting free vascular- ized fibular graft:}

- A normal preoperative arteriogram of bilateral lower extremities demonstrating three-vessel runoff of the right and left popliteal artery with normal patency of all distal vessels.

- The patient is placed in a supine position. The knee is flexed to $90^{\circ}$, and the hip is internally rotated with the use of a hip bump. The heel is then placed on a gel bumper, which is secured to the table. The patient is prepped and draped in a sterile fashion. A sterile tourniquet is applied

- Anatomic landmarks are palpated and marked to include the head of the fibula and the lateral malleolus of the ankle. The dorsalis pedis and posterior tibial pulses are palpated and marked with Doppler accordingly. With a surgical pen, a mark is placed $6-8 \mathrm{~cm}$ inferior to the head of the fibula and $6-8 \mathrm{~cm}$ superior to the lateral malleolus. This allows the proximal and distal $6-8 \mathrm{~cm}$ of the fibula and its ligamentus attachments to be preserved. A line is drawn along the posterior crural septum connecting the two marks.

- If a skin flap is to be harvested in conjunction with the fibula, the flap should be designed along the posterior septal crural. A Doppler will allow for the identification of septocutaneous perforating vessels

- Tourniquet inflated to $300 \mathrm{mmHg}$. Time of inflation must be recorded. Inflation time must be keep under 2 hours to avoid possible ischemic events.

- A skin incision is made to the depth of the superficial fascia along the length of the incision to include the skin flap.The peroneus longus muscle is elevated and retracted anteriorly and medially.

- Subperiosteal dissection is performed at the proposed proximal and distal osteotomy sites.
Protecting the medial aspect of the fibula and peroneal vessels, the proximal and distal osteotomies are performed with a reciprocating or oscillating saw, ensuring that minimum of $6-8 \mathrm{~cm}$ of fibula remains distal to the fibular head and proximal to the lateral malleolus.

- A bone clamp is used to retract the fibula laterally, allowing visualization of the peroneal vessels distall

- The dissection proceeds posterior laterally in the deep and superficial posterior compartments dividing the flexor hallucis longus and soleus muscles while maintaining a $5 \mathrm{~mm}$ muscle cuff on the fibula.

- Doppler is then used to confirm a strong signal of the peroneal vessels. The proximal peroneal vascular bundle is then ligated and divided. The fibula and skin paddle are passed to the reconstruction team.

- After adequate hemostasis is achieved, the deep muscle layers are reapproximated with 2-0 Vicryl interrupted sutures. Two suction drains are placed. The dermal layer is reapproximated. The skin is closed with staples or a nonresorbable suture. In the event that the skin flap is greater than $4 \mathrm{~cm}$, a split-thickness skin graft may be required.

Preparation of BMAC: according to Chahla et $\mathrm{Al}^{(19)}$

Fifty millimeters of autologous bone marrow was aspirated from the right anterior superior iliac spine. The aspiration was performed at 10 different spots on and around the anterior superior iliac spine, punctured by a 11-gauge vertebroplasty needle to get pure bone marrow ( $5 \mathrm{~mL}$ aspirated at each spot), because the aspiration of more than $10 \mathrm{~mL}$ at a single spot may raise the risk of collecting surrounding peripheral blood. A $60-\mathrm{mL}$ syringe already containing $10 \mathrm{~mL}$ anticoagulant citrate dextrose A was used. It is stable in the anticoagulated state for 8 hours. 


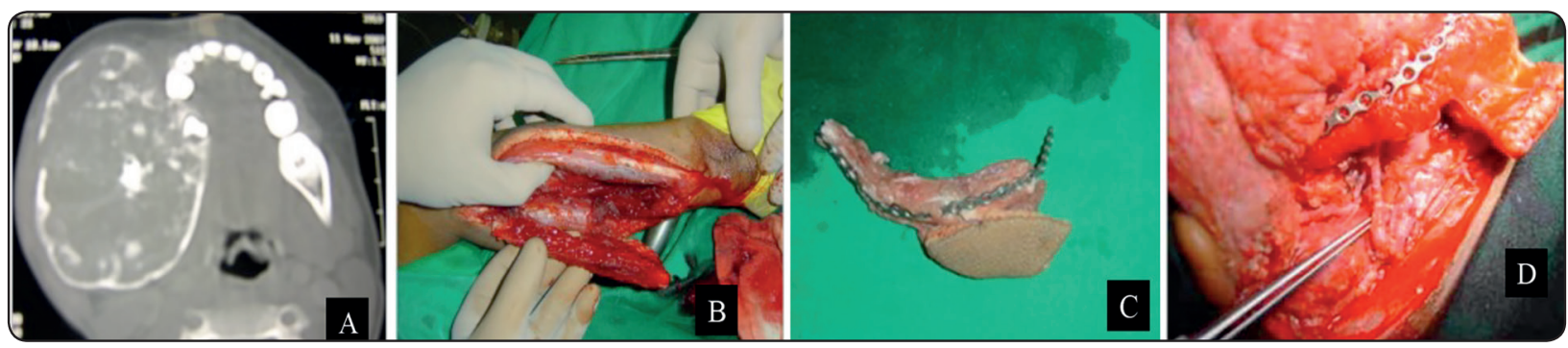

Fig. (1) Reconstruction of mandible after surgical removal massive fibrous dysplasia by free fibula flap.

\section{Processing of the Bone Marrow Aspirate:}

The bone marrow aspirate (BMA) sample must be processed after it is harvested. BMA is filtered through a 200 -mm-mesh filter into $50-\mathrm{mL}$ conical tubes. Then, 1 to $1.5 \mathrm{~mL}$ of the filtered BMA is pipetted into a 2-mL microcentrifuge tube for hemanalysis and the sample complete blood count with differential is automatically recorded. Subsequently, 60 to $90 \mathrm{~mL}$ of BMA is transferred into 2 50-mL conical tubes, and initially centrifuged at 2,400 rpm for 10 minutes. After completion of this process, the buffy coat layer and platelet-poor plasma layer are extracted from the conical tube and discarded. The red blood cell layers are combined into $150-\mathrm{mL}$ conical tube for second centrifugation $(3,400 \mathrm{rpm}$ for 6 minutes). Finally, the BMAC/white cell pellet is resuspended in platelet poor plasma, hemanalysis is performed and complete blood count with differential is recorded (including monocyte count) to assess the final product to inject

\section{Handling and Application of BMAC :}

The injection of $2 \mathrm{ml}$ of BMAC was conducted using a 22-gauge spinal needle for each injection. The injection was performed along site of reconstruction plate and iliac crest graft after closure of incision and slowly as possible to prevent possible washout by injection pressure (Fig. 2).

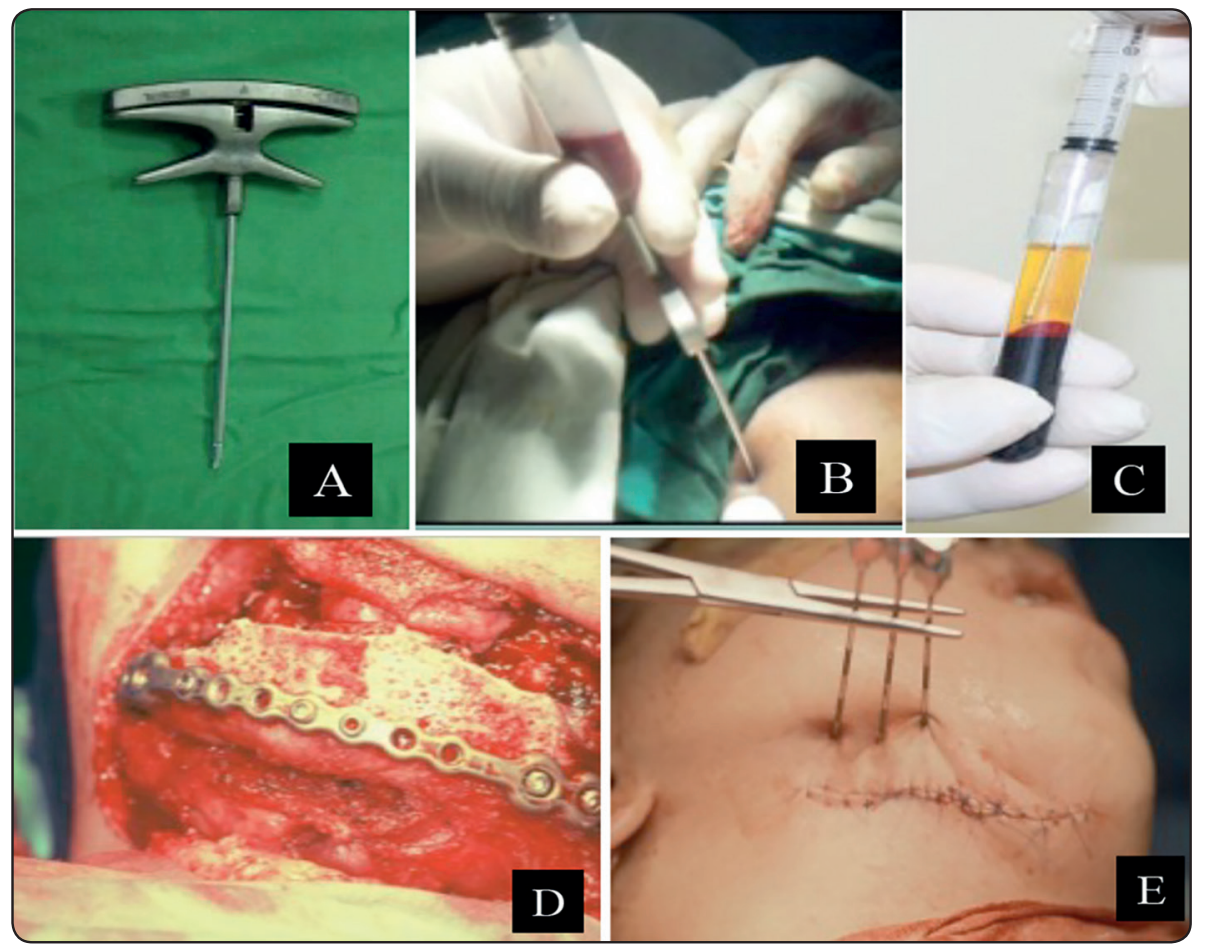

Fig. (2) a) Bone Marrow Aspiration Needle Set b) BM aspiration c) Aspiration of bone marrow concentration (clear zone) d) iliac crest bone graft fixed with reconstruction bone plate e) injection of BMAC 


\section{Postoperative care and follow-UP:}

\section{Postoperative care:}

Firstly, postoperative medications and instructions were described for patients to avoid postoperative complications such as infection, and edema. The patients were closely observed daily in the first week and then asked to attend for follow-up at $2^{\text {nd }}$, $3^{\text {rd }}$ and $4^{\text {th }}$ weeks and then after 6 months and one then two year postoperatively for clinical and radiographic evaluation.

\section{Postoperative clinical evaluation:}

Clinical evaluation involved observation of the following clinical parameters:

Wound examination for suture breakdown, dehiscence, swelling, infection, edema, hematoma, graft rejection, bone resorption, and recurrent of lesion.

\section{Postoperative radiographic assessment:}

Radiographic assessment postoperatively was done at the end of six and twelve month.Axial and coronal CT scans were made at about $0.5 \mathrm{~mm}$ section for each case to assess evidence of bone formation, presence of any bony bridging across the defect, density, and the height of the newly formed bone. Panoramic X-ray was used for follow up to assess recurrence after the first year.

\section{Bone density measurement}

Bone density was measured for each case on the axial cuts. The bone density was measured using Bioquant 15.1 software (BIOQUANT Image Analysis Corporation,www. bioquant. com), that measure average density for the target area. The bone density was measured for each patient at 6 and 12 month postoperatively.

\section{Statistical analysis}

Numerical data were presented as mean and standard deviation values. One-way ANOVA (Analysis of Variance) was used to compare between two groups. Duncan's post-hoc test was used for pair-wise comparison between the means when ANOVA test is significant. Paired t-test was used to study the changes by time in each group. The significance level was set at $P \leq 0.05$. Statistical analysis was performed with SPSS $17.0^{\circledR}$ (Statistical Package for Scientific Studies) for Windows.

\section{RESULTS}

Both groups was uneventful except one case $(14.3 \%)$ in group II showed dehiscence at incision site. Whom treated with drug prescription, vigorous saline irrigation and debridement in addition to patient instruction of good oral hygiene. Beside to 2 cases $(28.5 \%)$ in group I showed skin-paddle necrosis which needed revision surgeries after that. In group II , no injection-related adverse events were found, such as injection site pain or bone marrow aspiration site infection.

No recurrence of the disease was found by clinical or radiological examination ( fig.3) in both groups who were treated with complete excision of the mandibular lesions. The results showed that all patients had adequate facial contour, proper facial symmetry and good take of the free iliac crest graft and free fibula graft .

\section{Bone density:}

At six month the mean of group II was (174 pixels) less than the mean of group I (186 pixels). Therefore, there was a highly statistically significant difference between two groups $(p=0.01084)$. This means that, newly formed bone of group I was significantly denser than group II at the same interval. At 12 months, the mean of the group I was (245 pixels) more than the mean of group II (240 pixels). These showed a difference between means of two groups but this difference was not a statistically significant $(p=0.14250)$ (Table 1$) \&$ (fig.4). 


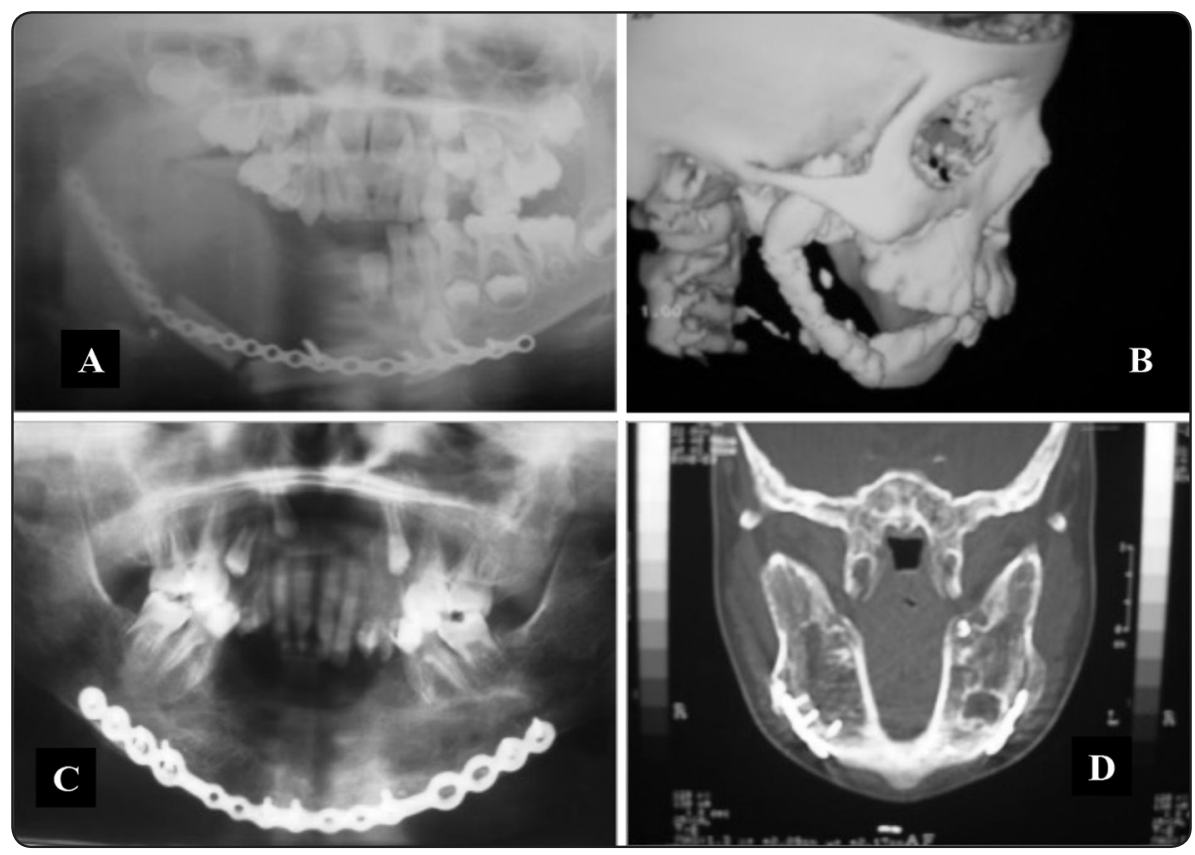

Fig. (3) A) panoramic x-ray showing fibula graft after 6 months in group I. B) Three Dimensions CT showing fibula graft after 12 months. C) panoramic x-ray showing iliac crest bone graft after 6 months in group II . D) Axial cut CT showing iliac crest bone graft after 12 months in group II.
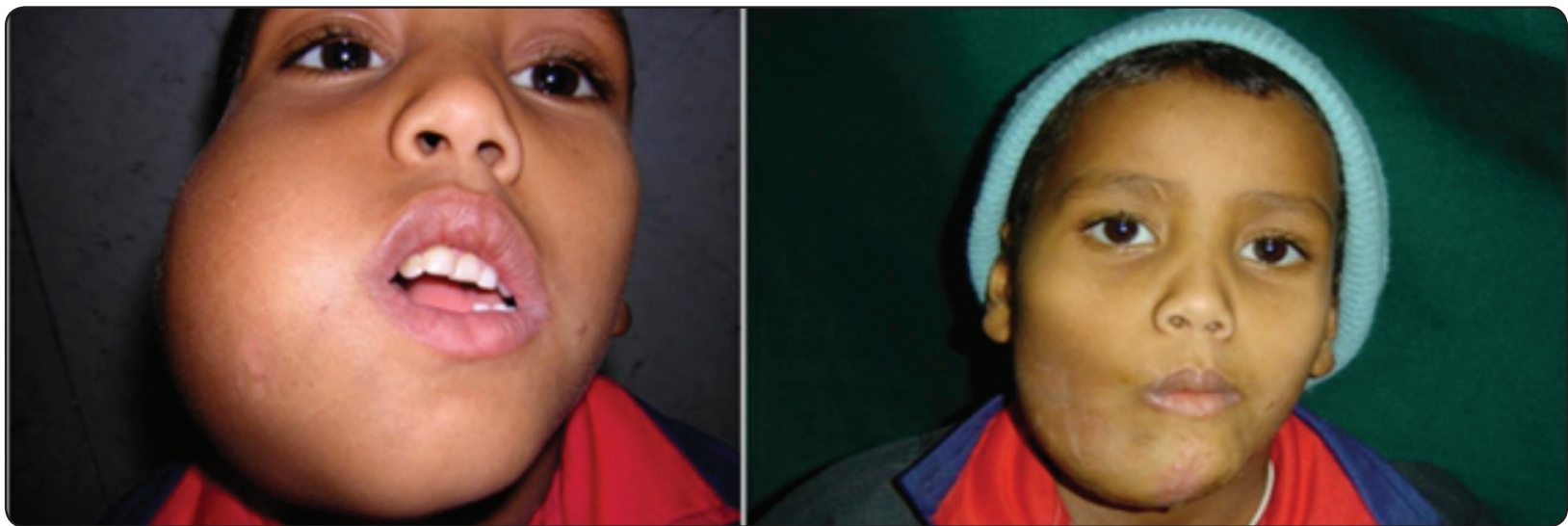

Fig. (4) Clinical pictures showing the case preoperative and postoperative after reconstruction with free vascularized fibular graft.

TABLE (1) Showing comparison of mean bone density of two groups at 6 and 12 month in addition to standard deviation (S.D) and $t$ - test for each interval and $p$ value

\begin{tabular}{|c|c|c|c|c|c|}
\hline \multicolumn{2}{|c|}{} & Mean & S.D & t -test & P \\
\hline \multirow{2}{*}{$6 \mathbf{~ m}$} & Group I & 186 & 7.440 & \multirow{2}{*}{2.965340} & \multirow{2}{*}{$0.01084^{*}$} \\
\cline { 2 - 4 } & Group II & 174 & 8.303 & & \\
\hline \hline \multirow{2}{*}{$\mathbf{1 2} \mathbf{~ m}$} & Group I & 245 & 12.95 & \multirow{2}{*}{1.71470} & \multirow{2}{*}{0.14250} \\
\cline { 2 - 4 } & Group II & 240 & 9.241 & & \\
\hline
\end{tabular}

* Significant at $\mathbf{P} \leq \mathbf{0 . 0 5}$,

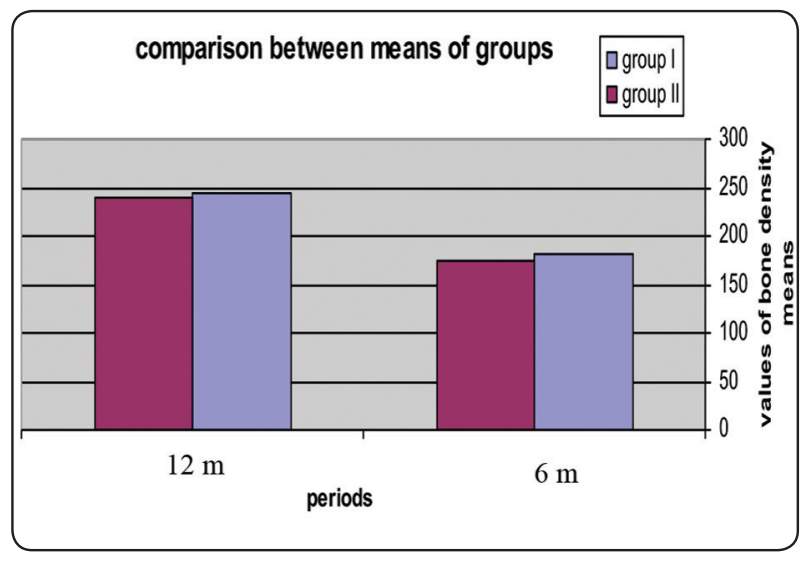

Fig. (5) Bar char demonstrating means of bone density at different intervals 


\section{DISCUSSION}

Fibrous dysplasia is a pathologic condition of bone of unknown etiology with no apparent familial, hereditary or congenital basis. Lichtenstein first coined the term in 1938 and in 1942 he and Jaffe separated it from other fibro-osseous lesions. It is a bone tumor that, although benign, has the potential to cause significant cosmetic and functional disturbance, particularly in the craniofacial skeleton. Its management poses significant challenges to the surgeon $^{(2)}$.

Surgical treatment of FD in maxillofacial region consists of either conservative shaving/contouring or radical excision with immediate reconstruction. Radical surgery followed by complex reconstruction techniques has been, at times, the treatment of choice for cases with associated complications such as neurological dysfunction, or growth retardation to preserve normal function and growth ${ }^{(20)}$.

The reconstruction of mandibular defects is complex due to the need to address both functional and aesthetic objectives. Several studies have noted that reconstruction with a free fibula bone graft has been shown to produce superior patient outcomes over NVBGs ${ }^{(12,13)}$. Nevertheless, it is also one of the most challenging procedures from the standpoint of craniomaxillofacial surgery. Recently, there has been a growing interest in biologic stimulations to enhance bone regenerate such as local application of bone marrow cells, platelet-rich plasma (PRP).

Studies ${ }^{(21,22)}$ implicates that bone marrowderived mesenchymal stem cells( BMSC) including progenitor cells of osteoblast, can be used to enhance angiogenesis and mineralization in bone defect. Bone marrow contains hematopoietic and nonhematopoietic stromal cells, from which osteoclasts and osteoblasts, respectively, originate. The inducible osteogenic precursor cells are capable of forming osteogenic tissue in the presence of inducers or stimulatory factors such as plateletderived growth factors ${ }^{(23,24)}$. So the use of BMAC might be of value in the promotion of healing in large bone defect. Depending on this suggestion
, the present study was preformed for evaluate efficacy of NVBGs with BMAC injection at bone defect versus VBG in monostatic fibrous dysplasia of mandible.

In the current study, radical excision was performed in all extensive mandibular swellings with simultaneous reconstruction by either iliac crest bone grafting or microvascular anastomosis free fibula transfer. This is in the same side with Ippolito et al ${ }^{(25)}$.

Cases with monostatic mandibular fibrous dysplasia were included in the present study due to they are most common occurred than other types. This in the same side with Ameli et al and Guruprasad et al ${ }^{(26,27)}$ study which concluded that The monostatic form of fibrous dysplasia is the most common, comprising $70 \%$ of cases, most likely to quiescent at puberty.

Present study showed that rate and volume of bone resorption after free non-vascularized bone grafting were in contrast with comparable results of Szpindor (28) and Lenzen C, et al ${ }^{(29)}$ studies which declared the patients with immediate reconstruction, more than $50 \%$ of the bone grafts resorbed. This can be explained by regenerative effect of BMAC on NVBG after immediate reconstruction of mandible which accelerate bone healing and decrease chance of infection occurrence. This matched with Chahla et $\mathrm{al}^{(19)}$ article.

In the present study $\mathrm{CT}$ radiographic assessment at six month revealed that in group II an evident newly formed bone with decrease in the density and arrangement of bone trabeculae in the center of the defects at this time. While, the group I at the same time showed adequate normal bone formation with adequate normal cortex and well arranged bone trabeculae in the center. In the same manner, at 12 month maturation in group I was more evident that in group II and the density was similar the normal adjacent bone at this time. This in the same direction with analysis data of denistromotric evaluation which showed no significant difference between both groups at 12 month. 


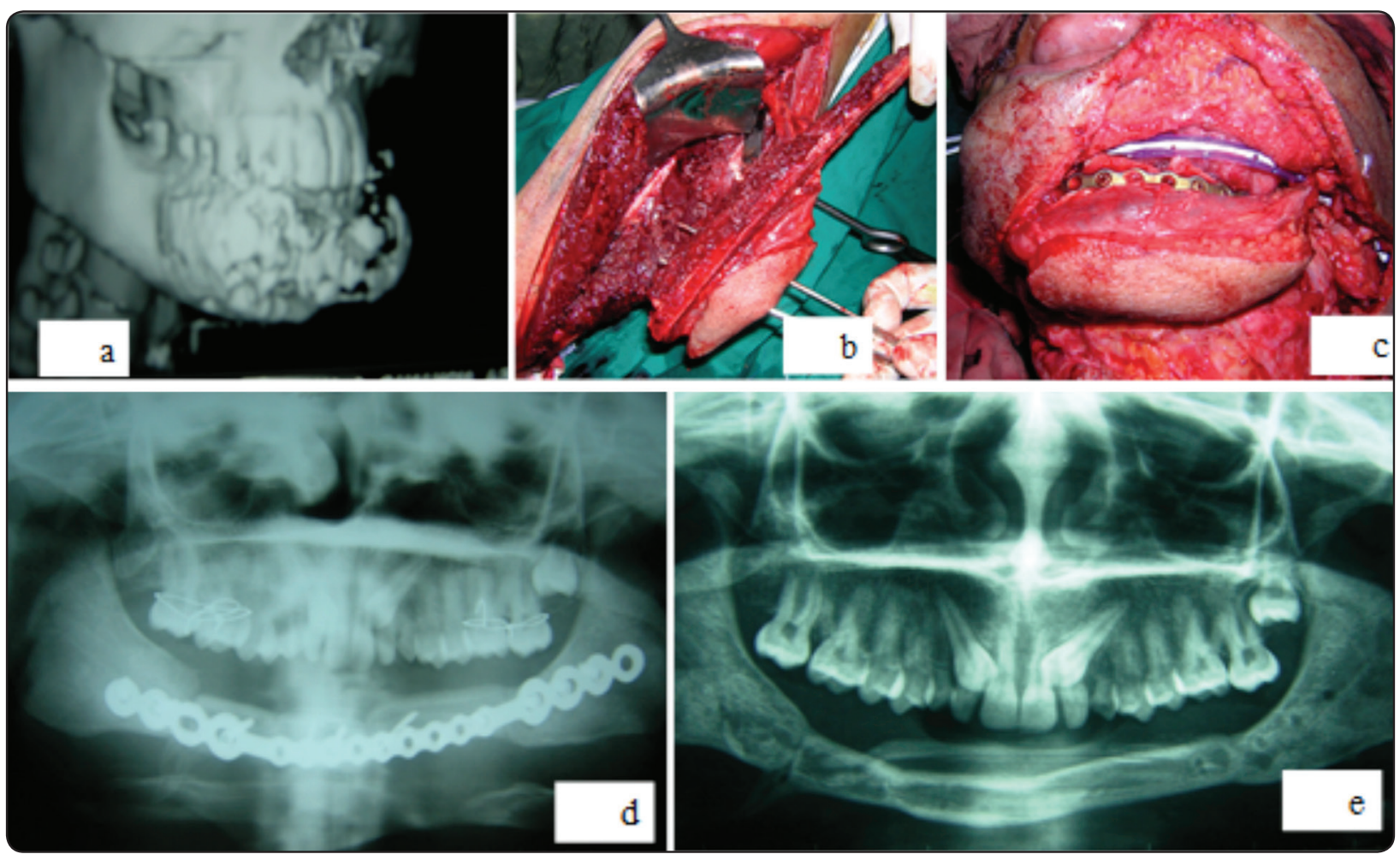

Case two : Reconstruction of mandible after surgical removal massive fibrous dysplasia by free fibula flap.

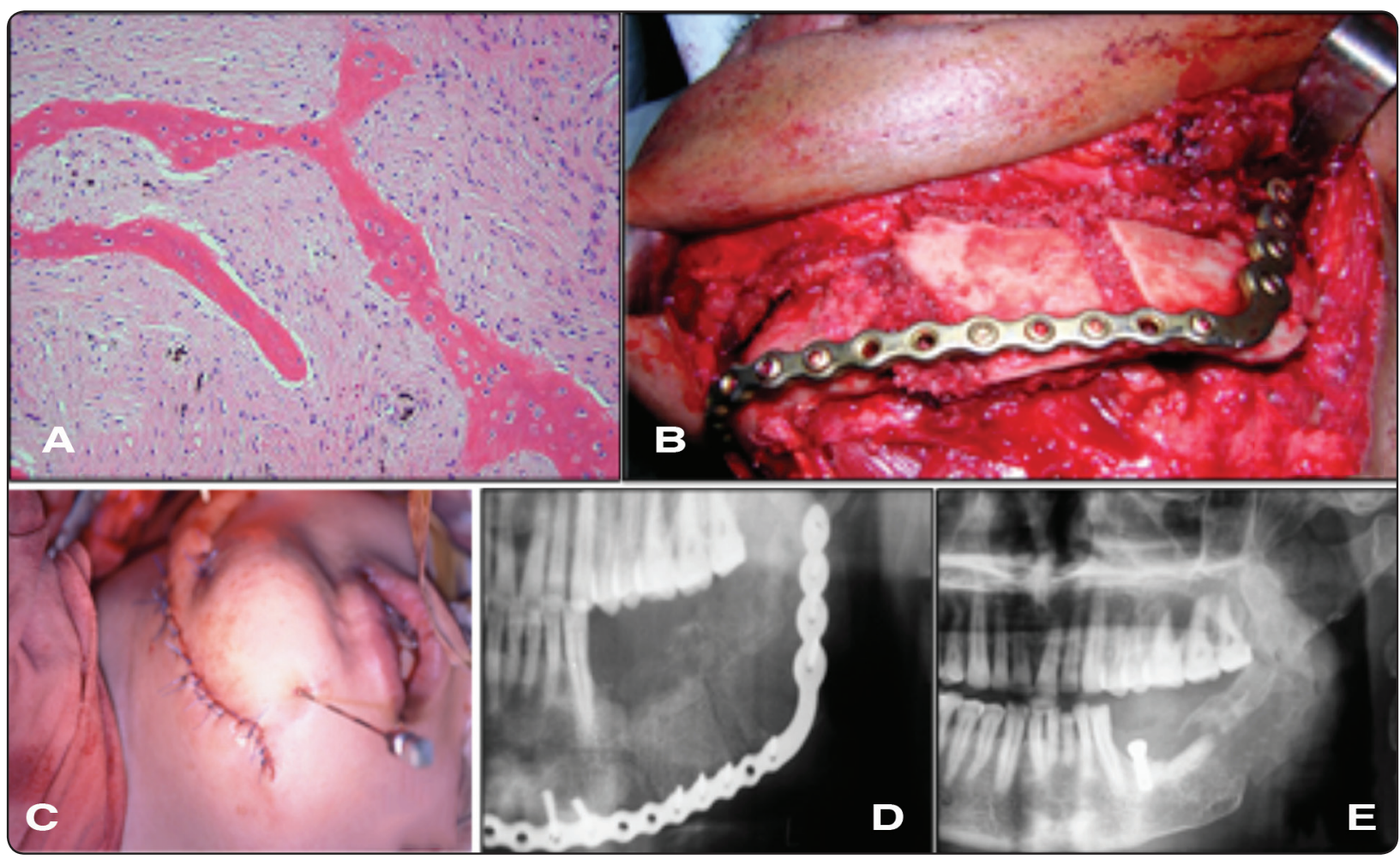

Case two(2) : Mandibular reconstruction after iliac crest bone graft fixed with reconstruction bone plate and injection of BMAC. 
Thereby, NVBG with BMAC injection may be a good alternative for free vascularized fibula due to no significant difference between them along time. This is in opposite side with Feingold et $\mathrm{al}^{(30)}$ statement. Who state on Microvascular free flap reconstruction has a role, especially for giant fbrous dysplasia involving the mandible where segmental excision is necessary.

\section{CONCLUSION}

Mandibular defect after surgical treatment of fibrous dysplasia should be reconstructed to function and cosmetics aims. Despite the imitated number of cases, results of the present study proved that the use of autogenous iliac bone graft with bone marrow aspirate concentrate injection has been shown to give a good result with little complications in our study, where facilities for free vascular fibula flap is not readily available.

\section{REFERENCES}

1. Yamamoto T, Ozono K, Kasayama S, Yoh K, Hiroshima $\mathrm{K}$, Takagi M,et al. Increased IL-6-production by cells isolated from the fibrous bone dysplasia tissues in patients with McCune-Albright syndrome. $J$ Clin Invest;98(1):30-5,1996.

2. DiCaprio M R, Enneking W F. Current concepts review. Fibrous dysplasia: Pathophysiology, evaluation, and treatment. J Bone Joint Surg (Am); 87 (8): 1848-64, 2005.

3. Ding C, Deng Z, Levine MA. A highly sensitive PCR method detects activating mutations of the GNAS1 gene in peripheral blood cells of patients with McCune-Albright syndrome or isolated fibrous dysplasia. J Bone Miner Res. 16(Suppl 1):S417, 2001.

4. Albright F, Butler AM, Hampton AO, Smith P. Syndrome characterized by osteitis fibrosa disseminata, areas of pigmentation and endocrine dysfunction, with precocious puberty in females. N Engl JMed 216:727-46,1937.

5. Hoshi M, Matsumoto S, Manabe J, Tanizawa T, Shigemitsu $\mathrm{T}$, Izawa N, et al. Malignant change secondary to fibrous dysplasia. Int J Clin Oncol;11(3):229-35, 2006.

6. Tehranzadeh J, Fung Y, Donohue M, Anavim A, Pribram HW. Computed tomography of Paget disease of the skull versus fibrous dysplasia. Skeletal Radiol 27:664-72, 1998.
7. Reymond J, Podsiadło M, Wyskiel M. Fibrous dysplasia situated inmaxilla-diagnostic and treatment difficulties illustrated with case report.Otolaryngol Pol;60:79-84, 2005.

8. Yetiser S, Gonul E, Tosun F, Tasar M, Hidir Y. Monostotic cra-niofacial fibrous dysplasia: the Turkish experience. $J$ Craniofac Surg 17:62-7, 2006.

9. Ralston SH. Pathogenesis of Paget's disease of bone. Bone;43:819-25, 2008.

10. Valentini V, Cassoni A, Marianetti TM, Terenzi V, Fadda MT, IannettiG. Craniomaxillofacial fibrous dysplasia: conservative treatment or rad-ical surgery? A retrospective study on 68 patients. Plast Reconstr Surg;123:653-60, 2009.

11. Maher CO, Friedman JA, Meyer FB, Lynch JJ, Unni K, Raffel C. Surgicaltreatment of fibrous dysplasia of the skull in children. Pediatr Neurosurg;37:87-92,2002.

12. Foster RD, Anthony JP, Sharma A, et al. Vascularized bone flaps versus nonvascularized bone grafts for mandibular reconstruction: an outcome analysis of primary bony union and endosseous implant success. Head Neck 21:66$71,1999$.

13. Pogrel MA, Podlesh S, Anthony JP, et al. A comparison of vascularized and nonvascularized bone grafts for reconstruction of mandibular continuity defects. J Oral Maxillofac Surg 55:1200-6,1997.

14. Kokosis g, Schmitz r, Powers d, Erdmann d. Mandibular Reconstruction Using the Free Vascularized Fibula Graft: An Overview of Different Modifications. Arch Plast Surg 43:3-9, 2016.

15. Chim H, Salgado CJ, Mardini S, et al. Reconstruction of mandibular defects. Semin Plast Surg;24:188-97, 2010.

16. Seto I, Marukawa E, Asahina I. Mandibular reconstruction using a combination graft of rhBMP-2 with bone marrow cells expanded in vitro. Plast Reconstr Surg 117:902-8, 2006.

17. Matsuo A, Chiba H, Takahashi H, et al. Bone quality of mandibles reconstructed with particulate cellular bone and marrow, and platelet-rich plasma. J Craniomaxillofac Surg 39:628-32, 2011.

18. Jager M, Herten M, Fochtmann $U$,et al. Bridging the Gap: Bone Marrow Aspiration Concentrate Reduces Autologous Bone Grafting in Osseous Defects J. Orthop. Res. 29:173-80,2011.

19. Chahla J, Mannava S, Cinque M, Geeslin A, Codina D, Laprade R. Bone Marrow Aspirate Concentrate Harvesting and Processing Technique. Arthroscopy Techniques, Vol 6, No 2 (April),: pp e441-e445,2017. 
20. Chen YR, Breidahl A, Chang CN. Optc nerve decompression in fbrous dysplasia: Indicatons, Efcacy, and Safety. Plast Reconstr Surg; 99:22-30, 1997.

21. Tsubota S, Tsuchiya H, Shinokawa Y, Tomita K, Minato H. Transplantation of osteoblast-like cells to the distracted callus in rabbits.J Bone Joint Surg 81B: 125-9,1999.

22. Mengchun Q, Jing H , Shujuan Z, Haixiao Z , Lichi H. Mandibular distraction osteogenesis enhanced by bone marrow mesenchymal stem cells in rats. Journal of Cranio-Maxillofacial Surgery 34, 283-9, 2006.

23. Pittenger M, Mackay A, Beck S, et al. Multilineage potential of adult human mesenchymal stem cells. Science 284: 143-7., 1999.

24. Quarto R, Mastrogiacomo M, Cancedda R, et al. Repair of large bone defects with the use of autologous bone marrow stromal cells. N Engl J Med 344: 385-8,2001.

25. Ippolito E, Caterini R, Farsetti P, Potenza V. Surgical treatment of fibrous dysplasia of bone in McCune-Albright syndrome. J Pediatr Endocrinol Metab. 15 Suppl 3:93944, 2002.

26. Ameli NO, Rahmat H, Abbassioun K. Monostotc fbrous dysplasia of the cranial bones: Report of fourteen cases. Neurosurg Rev 4:71-7, 1981.

27. Guruprasad Y, Chauhan DS. Craniofacial fibrous dysplasia - A review of current management techniques. Chron Young Sci; 3:106-10, 2012.

28. Szpindor E. Evaluation of the usefulness of autogenic bone grafts in reconstruction of the mandible. Ann Acad Med Stetin 41:155-69, 1995.

29. Lenzen C, Meiss A, Bull HG. Augmentation of the extremely atrophied maxilla and mandible by autologous calvarial bone transplantation. Mund Kiefer Gesichtschir 3(Suppl 1):S40-2, 1999.

30. Feingold RS, Argamaso RV, Strauch B. Free fbula flap mandible reconstructon for oral obstructon secondary to giant fbrous dysplasia. Plast Plast Reconstr Surg 97:196$201,1996$. 